\title{
Water Absorption Rate Prediction of PMMA and Its Composites Using BP Neural Network
}

\author{
Kui $\mathrm{CHEN}^{1, \mathrm{a}}$, Tianyun $\mathrm{ZHANG}^{2, \mathrm{~b}}$ and Shitang $\mathrm{BAO}^{2, \mathrm{c}}$ \\ ${ }^{1}$ School of Bailie Engineering \& Technology, Lanzhou City University, Lanzhou 730070, China \\ ${ }^{2}$ Center of Information and Network, Lanzhou City University, Lanzhou 730070, China

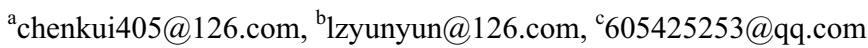

\begin{abstract}
Referring to water absorption rate of poly (methyl methacrylate) (PMMA) and its composites is hard to obtain under some working conditions, BP neural network prediction model was constructed. Regarding water absorption rate predictions of exfoliated PMMA/MMT nanocomposites in $0.1 \mathrm{~mol} / \mathrm{L} \mathrm{H}_{2} \mathrm{SO}_{4}$ solution, $0.1 \mathrm{~mol} / \mathrm{L} \mathrm{NaOH}$ solution and deionized water respectively as examples, the applicability of model established in water absorption rate prediction of PMMA and its composites was researched. The results show that the relative errors between prediction value obtained from model established and actual value of water absorption rate of composites soaking $63 \mathrm{~min}$ in three kinds of mediums are $1.50 \%, 0.47 \%$ and $1.04 \%$ respectively, prediction accuracy is higher than that (relative errors are $3.89 \%, 3.40 \%$ and $4.43 \%$ respectively) obtained from GM $(1,1)$ model obviously. BP neural network can be used to predict water absorption rate of PMMA and its composites.
\end{abstract}

Keywords: PMMA poly (methyl methacrylate); Water absorption rate; Prediction; BP neural network

\section{Introduction}

Poly (methyl methacrylate) (PMMA), also called organic glass, has excellent transparency, weather resistance, electrical insulation and processability. However, the existing of polar side-chain methyl leads to the fact that water can be absorbed by PMMA and its products [1], which may cause performances decline and even lead to deformation in severe cases, and affects safe use of PMMA and its products seriously [2]. Therefore, the data collection of water absorption rate is an important work before the application of PMMA and its products under some special conditions. Theoretically, this job can be done through experiments under artificial accelerated or actual working conditions. But there are inevitable differences between artificial accelerated condition and actual working condition, while experiments under actual working condition need a long time, may be several years, even decades. And it's difficult to keep up with the rapid development of materials research, let alone the cost of experiments is high [3]. Recently, according to experimental data related, forecasting properties of materials through mathematical model is one of the focus of attention and study [4].

One of the main points in mathematical modeling of forecasting for material properties 
is the nonlinear behavior of materials during aging. Artificial neural network, founded by McCulloch in the early 1940s, has emerged as a result of simulation of biological nervous system, such as the brain, on a computer [5]. The greatest advantage of artificial neural network is its ability to model complex non-linear, multi-dimensional function relationships without any prior assumptions about the nature of the relationships. Thus, taking the water absorption rate prediction of Poly (methyl methacrylate)/montmorillonite (PMMA/MMT) nanocomposites as an example, a BP (Back propagation) neural network approach was employed to predict the water absorption rate of PMMA and its composites in the presented paper. Furthermore, the prediction effect of the BP neural network model was compared to that obtained by the GM $(1,1)$ model.

\section{BP Neural Network Prediction Model}

BP neural network based on back propagation algorithm, one of the most well-known training algorithms for the multilayer perceptron, is one of the most widely used artificial neural networks [6]. The theory and practice show that a three-layer BP neural network can approximate any continuous function with arbitrary precision.

\subsection{The design of BP neural network}

Figure 1 is the structure of a BP neural network, including input layer, hidden layer and output layer. If there is $n$ training patterns, and each training pattern contains $m$ components, then the input layer contains $m$ nodes or neurons (corresponding to $m$ components of each training pattern). The input vector of each training pattern can be expressed as $\boldsymbol{X}=\left(x_{1}, \ldots\right.$, $\left.x_{i}, \ldots, x_{m}\right)^{\mathrm{T}}$ accordingly. Suppose the hidden layer has $k$ nodes, its output vector can be expressed as $\boldsymbol{Y}=\left(y_{1}, \ldots, y_{j}, \ldots, y_{k}\right)^{\mathrm{T}}$. Assume the output layer has only one node, then the output of $n$ training patterns can be expressed as $\boldsymbol{O}=\left(o_{1}, \ldots, o_{i}, \ldots, o_{n}\right)^{\mathrm{T}}$, and the desired output of $n$ training patterns, i.e. actual values, can be expressed as $\boldsymbol{D}=\left(d_{1}, \ldots, d_{i}, \ldots, d_{n}\right)^{\mathrm{T}}$, where $o_{i}$ and $d_{i}$ are the actual output and the desired output of the $i$ th training pattern respectively. The network-connection-weight (the quantitative expression of connected degree) between the input layer and the hidden layer can be expressed by matrix $\boldsymbol{V}=\left(v_{i j}\right)_{m \times k}$, where $v_{i j}$ is the network-connection-weight from the $i$ th node in the input layer to the $j$ th node in the hidden layer. The network-connection-weight vector between the hidden layer and the output layer is $\boldsymbol{Z}=\left(z_{1}, \ldots, z_{j}, \ldots, z_{k}\right)$, where $z_{j}$ is the network-connection-weight from the $j$ th node in the hidden layer to the output layer.

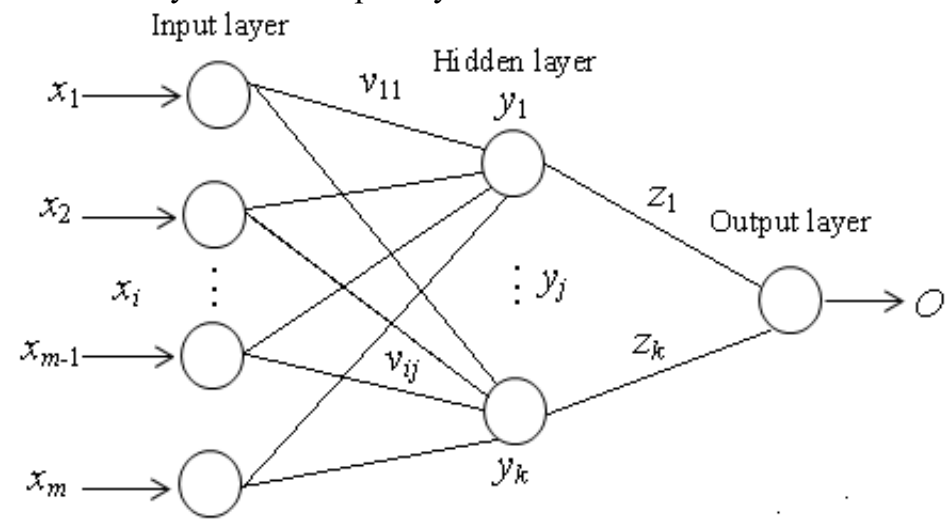

Fig.1 BP neural network structure

The main duty of the BP artificial neural network design is to determine the numbers of node in each layer. The practice indicates that, the minimum error of prediction simulation 
can be obtained when the node number of hidden layer is three [7]. The node number of the input layer was taken four as a result of trying different number of nodes, while that of the output layer was taken one since only water absorption rate need to predict. Thus, a BP neural network structure of $(4,3,1)$ was selected in the presented paper, i.e., there were four, three and one nodes in the input, hidden and output layer respectively.

\subsection{The calculation of output values}

The net output of the output layer and the hidden layer are calculated by using Eqs. (1) and (2) respectively.

$$
\begin{gathered}
O=f\left(\sum_{j=1}^{k} z_{j} y_{j}\right) \\
y_{j}=f\left(\sum_{i=1}^{m} v_{i j} x_{i}\right) \quad j=1,2, \ldots, k
\end{gathered}
$$

Activation function is a function that processes the net input obtained from sum function and determines the cell output. The most common activation functions are ramp, sigmoid, and Gaussian function. In the present study, a sigmoid function given in Eq. (3) is employed as the activation function in the training of the network.

$$
f(x)=\frac{1}{1+e^{-x}}
$$

\subsection{Network training}

The constructing of the training patterns is determined by experimental data given. The network-connection-weights of matrices $\boldsymbol{V}$ and $\boldsymbol{Z}$ are assigned a smaller value between 0 and 1 randomly. Then data of the first training pattern are regarded as the net input of $m$ nodes in the input layer. The network training is performed as the following steps.

(1) The calculation of network error. When the net output $o_{i}$ of the $i$ th training pattern is unequal to the desired output $d_{i}$, the output error $E_{i}$ of the $i$ th training pattern is calculated as:

$$
E_{i}=\frac{1}{2\left(d_{i}-o_{i}\right)} \quad i=1,2, \ldots, n
$$

And the total error $E$ of the network is determined as below:

$$
E=\frac{1}{2} \sum_{i=1}^{n} E_{i}
$$

(2) The adjusting of weights. Weights are values that express the effect of an input set or another process element in the previous layer on this process element. For the $i$ th training pattern, the error information $\delta^{o}$ of the output layer is obtained by Eq. (6) through comparing target output $d_{i}$ with actual output $o_{i}$ of the output layer. When the error information $\delta^{o}$ is processed in the backward from the output layer to the hidden layer, the error information $\delta^{y}$ of the hidden layer is obtained by Eq. (7). Then the network-connection-weights between the hidden layer and the output layer are adjusted by Eq. (8), and that between the input layer and the hidden layer are adjusted by Eq. (9).

$$
\begin{array}{r}
\delta^{o}=\left(d_{i}-o_{i}\right) o_{i}\left(1-o_{i}\right) \\
\delta^{y}=\delta^{o} z_{j} y_{j}\left(1-y_{j}\right)
\end{array}
$$




$$
\begin{aligned}
& z_{j}(t)=z_{j}(t-1)+\eta \delta^{o} y_{j}+\mu \Delta z_{j}(t-1) \\
& v_{i j}(t)=v_{i j}(t-1)+\eta \delta^{y} x_{i}+\mu \Delta v_{i j}(t-1)
\end{aligned}
$$

where $\eta(0<\eta<1)$ is the learning rate, $\mu$ is the momentum term, usually taken between 0 and $1, t$ is current training, $t-1$ is precious training, $\Delta$ is the adjustment value of network-connection-weight correspondingly. The momentum term $\mu$ reflects the adjustment of experience before, and can accelerate the convergence speed of the network, i.e., improving training speed of network.

(3) Inputting the next training pattern and returning to step (1).

(4) The finishing of network training. After all the training patterns have been presented, training procedure can be ceased on condition that the total error $E$ is less than a selected accuracy $E_{\min }$ (usually set as a positive decimal) of network training. Otherwise $E$ is assigned as 0 , return to step (1) and retrain the network from the first pattern again.

\subsection{Network prediction}

The BP neural network can perform prediction when its training meets the requirement of accuracy, i.e. $E<E_{\min }$. Prediction value can be obtained when the network accepts the new input parameters accordingly.

\section{Applicability Research of Prediction Model}

Taking the water absorption rate predictions of PMMA/MMT nanocomposites soaked in acid, base and deionized water as examples, the applicability of the BP neural network prediction model established above in water absorption rate prediction of PMMA and its composites was researched.

A small amount of MMT dispersed evenly in the PMMA matrix in nanoscale lamella disorderly increases effective way of water diffusion movement on PMMA matrix, and improves liquid barrier property of matrix. Accordingly, water absorption rate of composites reduces obviously. Table 1 is the relationships between the soaking time and the water absorption rate of exfoliated PMMA/MMT nanocomposites containing 3wt\% MMT soaked in $0.1 \mathrm{~mol} / \mathrm{L} \mathrm{H}_{2} \mathrm{SO}_{4}$ solution, $0.1 \mathrm{~mol} / \mathrm{L} \mathrm{NaOH}$ solution and deionized water respectively [8].

To predict water absorption rate of composites after soaking 63 minutes in $\mathrm{H}_{2} \mathrm{SO}_{4}$ solution, the training patterns of the $\mathrm{BP}$ neural network were constructed according to that of composites after soaking 7 to 56 minutes in $\mathrm{H}_{2} \mathrm{SO}_{4}$ solution, as seen in Table 2 .

As previously mentioned, the number of nodes in the input, hidden and output layer of the BP neural network were taken four, three and one respectively. The learning rate $\eta$ was taken 0.6 , the momentum term $\mu$ was taken 0.6 , and the network training accuracy $E_{\min }$ was determined as 0.001 . The $\mathrm{BP}$ neural network algorithm written in $\mathrm{C}++$ was trained with 8 sets of experimental data consisting of input and output values in Table 2 . 
TAB.1 EFFECT OF SOAKING TIME ON WATER ABSORPTION RATE OF PMMA/MMT NANOCOMPOSITES

\begin{tabular}{cccc}
\hline \multirow{2}{*}{$\begin{array}{c}\text { Soaking } \\
\text { time } / \text { min }\end{array}$} & \multicolumn{3}{c}{ Water absorption rate } \\
\cline { 2 - 4 } & $\mathrm{H}_{2} \mathrm{SO}_{4}$ solution & $\mathrm{NaOH}$ solution & Deionized water \\
\hline 7 & 0.5036 & 0.6241 & 0.4023 \\
14 & 0.6224 & 0.7430 & 0.5212 \\
21 & 0.7128 & 0.8334 & 0.6112 \\
28 & 0.7623 & 0.8777 & 0.6553 \\
35 & 0.8019 & 0.9220 & 0.7002 \\
42 & 0.8473 & 0.9678 & 0.7456 \\
49 & 0.8762 & 0.9965 & 0.7753 \\
56 & 0.9019 & 1.0229 & 0.7999 \\
63 & 0.9125 & 1.0332 & 0.8110 \\
\hline
\end{tabular}

TAB.2 TRAINING PATTERNS

\begin{tabular}{cccccc}
\hline Sample number & \multicolumn{5}{c}{ Input } \\
\hline 1 & 0 & 0 & 0 & 0 & Output \\
2 & 0 & 0 & 0 & 0.5036 & 0.6224 \\
3 & 0 & 0 & 0.5036 & 0.6224 & 0.7128 \\
4 & 0 & 0.5036 & 0.6224 & 0.7128 & 0.7623 \\
5 & 0.5036 & 0.6224 & 0.7128 & 0.7623 & 0.8019 \\
6 & 0.6224 & 0.7128 & 0.7623 & 0.8019 & 0.8473 \\
7 & 0.7128 & 0.7623 & 0.8019 & 0.8473 & 0.8762 \\
8 & 0.7623 & 0.8019 & 0.8473 & 0.8762 & 0.9019 \\
\hline
\end{tabular}

After the training of the network, the water absorption rate of composites soaking 63 minutes in $0.1 \mathrm{~mol} / \mathrm{L} \mathrm{H}_{2} \mathrm{SO}_{4}$ was predicted, the result was given in Table 3. Table 3 also gave the prediction values of the water absorption rate of composites after soaking 63 minutes in $0.1 \mathrm{~mol} / \mathrm{L} \mathrm{NaOH}$ and deionized water respectively. The detailed calculating processes were omitted here (Since both the input and output of water absorption rate of composites soaked in $\mathrm{NaOH}$ solution were divided by 10 to acquire a value between 0 and 1 , the network training accuracy $E_{\min }$ was determined as 0.0001 , and the output should be multiplied by 10 to restore).

TAB.3 PREDICTION RESULTS

\begin{tabular}{cccccc}
\hline Soaking medium & \multicolumn{3}{c}{ Input } & Prediction value \\
\hline $\mathrm{H}_{2} \mathrm{SO}_{4}$ solution & 0.8019 & 0.8473 & 0.8762 & 0.9019 & 0.8987 \\
$\mathrm{NaOH}$ solution & 0.9220 & 0.9678 & 0.9965 & 1.0229 & 1.0283 \\
Deionized water & 0.7002 & 0.7456 & 0.7753 & 0.7999 & 0.8025 \\
\hline
\end{tabular}

Relative errors between prediction values and actual values of water absorption rate were given in Table 4. Table 4 also gave the prediction results and relative errors obtained by the GM $(1,1)$ model based on Grey Theory [8].

TAB. 4 RELATIVE ERRORS OF PREDICTION RESULT BASED ON BP NEURAL NETWORK MODEL

\begin{tabular}{lllccc}
\hline & & \multicolumn{2}{c}{ BP neural network model } & \multicolumn{2}{c}{ GM $(1,1)$ model } \\
\cline { 3 - 6 } Soaking medium & Actual value & Prediction value & $\begin{array}{c}\text { Relative } \\
\text { error }\end{array}$ & $\begin{array}{c}\text { Predicti } \\
\text { on } \\
\text { value }\end{array}$ & $\begin{array}{c}\text { Relative } \\
\text { error }\end{array}$ \\
\cline { 3 - 6 } & & & $1.50 \%$ & 0.9480 & $3.89 \%$ \\
$\mathrm{H}_{2} \mathrm{SO}_{4}$ solution & 0.9125 & 0.8987 & $0.47 \%$ & 1.0683 & $3.40 \%$ \\
$\mathrm{NaOH}_{\text {DaOlution }}$ & 1.0332 & 1.0283 & $1.04 \%$ & 0.8469 & $4.43 \%$ \\
\hline
\end{tabular}


It can be seen from Table 4 that relative errors of the water absorption rate between prediction value obtained from the BP neural network prediction model and actual value of composites after soaking 63 minutes in three kinds of mediums are $1.50 \%, 0.47 \%$ and $1.04 \%$ respectively, which not only are allowable for industrial application, but also are lower than those obtained from the GM $(1,1)$ model obviously. The results above show that prediction results obtained from BP neural network are reasonable and reliable. And BP neural network can be used to predict water absorption rate of PMMA and its composites.

Essentially, input and output problem of patterns is converted into a nonlinear optimization problem by BP neural network through the using of one of the most common gradient descent algorithm in the optimization technology. The solving of water absorption rate through iterative operation is equal to the problem of learning and memory. A scientific, reasonable and practical knowledge checked by practice can be obtained by BP neural network through the study of known patterns. Thus, neural network can avoid the disadvantageous factors of other prediction methods and guarantee the effectiveness and practicability of the prediction results.

There's no doubt about the advantages mentioned above, but it should be attention that training patterns are the base of neural network prediction. The quality of the training patterns is crucial to predictive effect. The training patterns adopted by the neural network must meet large quantity and wide coverage. Therefore, realizing water absorption rate prediction of PMMA and its composites by using BP neural network algorithm is totally feasible in theory, but when there are no enough training patterns or the deviation of the soaking time and that of the coverage area of sample data is severe, prediction accuracy will reduce correspondingly.

\section{Conclusions}

Referring to the question that under some working conditions, water absorption rates of PMMA and its composites are hard to obtain, the BP neural network prediction model was constructed. The water absorption rate prediction of exfoliated PMMA/MMT nanocomposites soaked in $0.1 \mathrm{~mol} / \mathrm{L} \mathrm{H}_{2} \mathrm{SO}_{4}$ solution, $0.1 \mathrm{~mol} / \mathrm{L} \mathrm{NaOH}$ solution and deionized water respectively shows that the maximum relative error of water absorption rate between prediction value obtained from the BP neural network prediction model constructed and actual value in three kinds of mediums is $1.50 \%$, lower than that obtained from the GM $(1,1)$ model obviously. The BP neural network is a useful tool in characterizing the influence of soaking time on water absorption rate of PMMA and its composites if especially a sufficient amount and wide coverage of experimental data is obtained.

\section{Acknowledgement}

This research was supported by No. LZCU-XZ2014-14 of the Scientific Research Foundation of Lanzhou City University and No. 1111B-03 of the Scientific Research Project of Bureau of Gansu Education.

\section{References}

1. G. H. Kim, A PMMA composite as an optical diffuser in a liquid crystal display backlighting unit (BLU), European Polymer Journal. 41(2005) 1729-1737. 
2. $\mathrm{M} . \mathrm{Wu}$, The study on residual stress testing of humidity effect and distribution law for PMMA, Advanced Materials Research. 631-632 (2012) 358-361.

3. X. Colin, L. Audouin, J. Verdu, M. Rozental-Evesque, B. Rabaud, F. Martin, F. Bourgine, Aging of polyethylene pipes transporting drinking water disinfected by chlorine dioxide. Part II-Lifetime prediction, Polymer Engineering \& Science. 49 (2009) 1429-1437.

4. Ever J. Barbero, Prediction of long-term creep of composites from doubly-shifted polymer creep data, Journal of Composite Materials. 43 (2012) 2109-2124.

5. M. Yilmaz, E. H. Metin, The prediction of mechanical behavior for steel wires and cord materials using neural networks, Materials and Design. 28 (2007) 599-608.

6. X. F. Cao, A BP neural network meteorological prediction, Bulletin of Science and Technology. 28 (2012) 55-57.

7. Y. He, X. L. Li, X. F. Deng, Discrimination of varieties of tea using near infrared spectroscopy by principal component analysis and BP model, Journal of Food Engineering. 79 (2007) 1238-1242.

8. K. Chen, T. Y. Zhang, S. C. Liu, X. P. Zhen, Application of improved grey prediction model on water absorption rate prediction of PMMA and its composites, Engineering Plastics Application. 41 (2013) 76-79. 